\title{
Modernization of grinding machines of grain cleaning machines
}

\author{
Aleksey Dorokhov ${ }^{1}$, Victor Khamyev ${ }^{1, *}$, and Kirill Lepeshkin ${ }^{1}$ \\ ${ }^{1}$ Federal Scientific Agro-Engineering Center VIM, 1 Institutsky proezd, 5, Moscow, 109428, Russia
}

\begin{abstract}
Annotation. As a result of the analysis of developed earlier mathematical models of the process of seeds purification on a two-tier lattice mill of polymeric materials the possibility of significant improvement in the quality of seed treatment and performance at the same quality at the expense of more rational technological schemes and modernization of the sieve camp's were revealed. Constructive changes were made in the lattice mill with using a new type of polymer sieves. Result of this experimental studies confirm the identified possibility of intensification of the process of seed cleaning - completeness of waste allocation increased from 0,653 to 0.75 , i.e. $15 \%$, performance for the same quality of cleaning have increased by $24 \%$.
\end{abstract}

\section{Introduction}

It is planned research work in Federal Scientific Agro-Engineering Center VIM to create a secondary machine of seed cleaning capacity of $20 \mathrm{t} / \mathrm{h}$. the new machine is developed on the basis of production machine ZVS-20, which has a capacity of $20 \mathrm{t} / \mathrm{h}$ for cleaning grains. Requirements to indicators of quality of seeds cleaning are significantly higher, than grain cleaning, so it is necessary to find ways to intensify and determine the parameters of the main working body of the machine-lattice separator.

\section{Materials and methods}

We determined the quality parameters of seed cleaning (the completeness of separation of waste) on two-tier lattice mill with two placement schemes sieve in the mill - known, applied in production machines and new (experimental).

The experimental scheme of the sieve is made of polymer material UHMW PE[1,2,3]

The studies were carried out during the feed of the source material in the kinematic mode of movement of the sieve mill made of polymeric materials and other factors, suitable for machine ZVS-20A work.

Experiments were carried out on a laboratory facility designed for these objectives and described earlier in the scientific report of the laboratory. The investigated schemes of the lattice mill are presented in Fig. 1 and 2.

\footnotetext{
* Corresponding author: victor250476@yandex.ru
} 


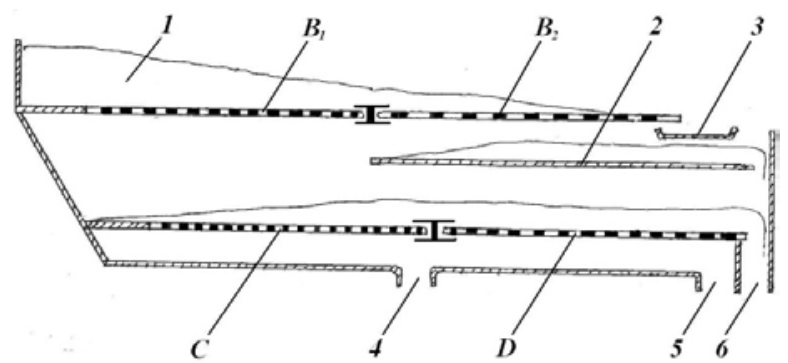

1 - processed material; 2 - grain board; 3 - large-scale waste receiver; 4 - receiver of sowing; 5 small waste receiver; 6 - receiver is clean of seeds

Fig. 1. Diagram of production sieve mill.

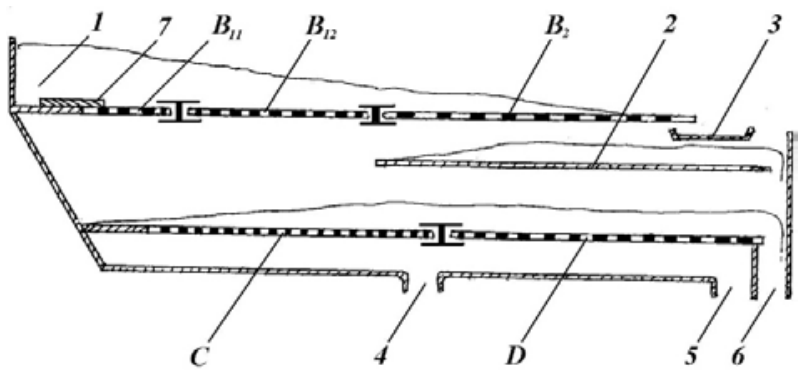

1 - processed material; 2 - grain board; 3 - large-scale waste receiver; 4 - receiver of sowing; 5 small waste receiver; 6 - receiver is clean of seeds; 7 - sheet length adjustment of the polymer sieve $B_{11}$

Fig. 2. Diagram of an experimental lattice mill with polymer sieves.

Polymer sieve pan production (Fig.1) contains four sieve of the same length, placed in two tiers. Hole size of the first sieve B1, called discharge, is selected so that through it approximately half of the seed input was sifted. The rest of the material goes to the sieve B2, the holes of which are seeds of the main crop. Seeds that have passed through the sieve B2 are considered to be cleaned from minor and major impurities.

Part of the seed that passes through the sieve B1 falls on the sowing sieve C, serving to separate small waste, and then descends to grading sieve $\mathrm{D}$, where seeds are cleaned from a larger waste (small, fine, crushed seeds and other impurities). Material coming down from the sieve $\mathrm{D}$ is considered to be cleared and seeds combined with the seeds passed through sieve B2 (right off the transport Board).

Considered sieve mill ensures the required quality of cleaning condition that the thickness of the layer of the processed material through the sieve does not exceed a certain value, depending on the feed material and its speed move through the sieve.

If the thickness of the layer on the B1 is higher than the critical level, the part of the seed material comes down to the sieve B2 together with the small waste, which further not allocated, as a result, the efficiency of seed cleaning is drastically reduced.

If the number coming down from the sieve B1 material decrease due to the increase of passage through this sieve then the thickness of the layer on the sieves $C$ and D increases and then these sieves do not provide the required completeness of fine waste separation.

Polymer sieve B1 does not provide a rational distribution of the treatment - the melted material in the sieve mill with increased flows. At first half of sieve B1 the fine waste is separated only from the part of the material layer due to large his thickness. To separate fine particles from the upper layers of the material remains the second half of the sieve B1, which is not enough to achieve the required quality of cleaning. At the same time, the first 
half of sieve $\mathrm{C}$ is not loaded with material, since this area receives no more than a fourth of all materials.

Analysis of the work of the lattice mill revealed the possibility of intensification seed cleaning process due to more efficient distribution of processed material on the lattices of the mill and change the function of the sieve B1.

In the experimental scheme (Fig.2.) polymer sieve B1 is made by composite of the two solvers of the same total length as B1. The first sieve B11 has holes of the same size as the lattice B2 and is used to separate the incoming of lattice mill of the material into two streams of approximately equal power. Such separation is provided by a choice of appropriate length of the sieve and may be regulated by changing it by covering the initial section of the sieve by metal sheet.

The second sieve B12 is designed to clean the incoming seeds from small waste. It has holes of the same size as the grading sieve D. the working length of the sieve B11 is 100$200 \mathrm{~mm}$, the length of the sieve B12-800 mm.

The lower tier of the sieves of the pilot mill is not different from production.

Seed cleaning on the experimental machine is carried out as follows. Entering the sieve pan seed sieve B11 is divided into two roughly equal parts. The half of the material passed through the sieve B11 arrives at the beginning of the sieve from the lower tier, cleaned on it and then on the sorting sieve $G$ from minor impurities. Some of the material of the sieve B11 gets to sorting sieve B12, it is cleaned of fine impurities and then comes to a sieve B2, where large impurities stand out.

The waste that passes through the sieve B12 falls on the sieve $\mathrm{C}$ and then on the sieve D and the passage stands out to them.

The process of separation of the seed material on the experimental polymer of the sieve mill should be more efficient than the production mill since in this case the grating along the entire length has the best loading, which can be obtained at a given feed material.

Research was carried out on the seed material of wheat. Source material was pre-cleared on pneumocephalus device and analyzed on the sieve classifier in thickness (on the sieve with oblong holes.) The distribution of material by thickness of particles is presented in the table 1 .

Based on the data of table 1 on the lattice mill, diagram Fig. I have installed the following sieves: $\mathrm{B} 1-2,8 \times 25$; B2 $-3,5 \times 25$; $\mathrm{C}$ is $1.8 \times 25$; D is $2.2 \times 25$.

When using the sieve $\mathrm{D}$ with holes $2,2 \mathrm{~mm}$ wide, the content of the fine waste is equal to $4.01 \%$. The width of the holes of the installed sieve B1 and $2.8 \mathrm{~mm}$, according to table 1 , should provide sifting through it about half of materials. However, as shown by the experiments of the installation series, the passage of this sieve only $19.8 \%$ of the material is released, so it was replaced with a sieve with holes with a width of $3 \mathrm{~mm}$. The throughput of the sieve B2 $(3,5 \times 25)$ also turned out to be insufficient in this regard has established a sieve with openings of a width $4.0 \mathrm{~mm}$. The quantity of seed material used in the experiments was $23.5 \mathrm{~kg}$.

Table 1. Distribution of seed material by particle thickness

\begin{tabular}{|l|c|c|c|c|c|c|c|c|c|}
\hline $\begin{array}{l}\text { Thickness of } \\
\text { particles, mm }\end{array}$ & $0-1,5$ & $1,5-1,8$ & $1,8-2,0$ & $2,0-2,2$ & $2,2-2,4$ & $2,4-2,6$ & $2,6-2,8$ & $2,8-3,0$ & $3,0-3,4$ \\
\hline $\begin{array}{l}\text { Amount of mate- } \\
\text { rial in \% by } \\
\text { weight }\end{array}$ & 0,88 & 1,37 & 0,68 & 1,08 & 7,14 & 28,04 & 24,28 & 24,13 & 12,4 \\
\hline
\end{tabular}

Experiments were carried out in the following sequence. The source material after thorough mixing was loaded into the hopper feeder. We turned on the drive sieve pan, then feeder and at the same time included a stopwatch to control the duration of flow materials. After processing all the loaded material produced weighing of all fractions and analysis of 
purified seeds. The contents of waste in the cleaned material were determined by recleaning it on the grating to termination of withdrawal. The presentation of the material was determined and controlled by the time of his passes.

According to the results of the analysis of fractions we calculated the completeness of the separation of the upper and lower tiers.

\section{Results and discussion}

The results of the experiments are presented in table 2-3.

Analysis of the obtained materials shows that the new (fractional) scheme of the modernized grating mill made of polymeric materials provides more effective seed cleaning in comparison with a standard one. The completeness of separation small waste, allocated seeding and grading sieves, increases with 0.65 to 0.75 at the level of the accelerations of $15.2 \mathrm{~m} / \mathrm{S} 2$ and from 0.71 to 0.80 with the level of accelerations $18.9 \mathrm{~m} / \mathrm{S} 2$.

Productivity of the new grating mill is $24 \%$ higher than that of the production with the same efficiency of seed cleaning.

The low efficiency of the production lattice mill is explained by the fact that the sieve B1 does not provide a sufficiently high completeness of discharge from the material coming from this sieve. Completeness of discharge by lower tier of the sieve reaches 0.86 , and the top - only $0.62 \ldots 0,65$.

The highest efficiency of seed treatment on experimental lattice of the mill is reached at such distribution of material when on the bottom tier $60-65 \%$ of the material is passed, and the rest is processed on the top.

The increased level of accelerations of vibrations of the sieve mill from 15.2 to 18.9 $\mathrm{m} / \mathrm{S} 2$ (which accords in the car ZVS-20A levels frequency 430 and $480 \mathrm{~min}-1$ ) increases the completeness of separation of waste both in manufacturing and experimental sieve mills by $5 \%$.

Table 2. Results of experiments on cleaning wheat seeds

\begin{tabular}{|c|c|c|c|c|c|}
\hline \multirow{2}{*}{$\begin{array}{c}\text { №No } \\
\text { pp }\end{array}$} & $\begin{array}{c}\text { scheme of the lattice } \\
\text { mill }\end{array}$ & $\begin{array}{c}\text { width of sieve } \\
\text { holes } \mathrm{B}_{1} \\
\left(\mathrm{~B}_{11} \mathrm{~B}_{12}\right), \mathrm{mm}\end{array}$ & $\begin{array}{c}\text { sieve length } \mathrm{B}_{1} \\
\left(\mathrm{~B}_{11} / \mathrm{B}_{12}\right), \mathrm{mm}\end{array}$ & $\begin{array}{c}\text { acceleration } \\
\text { level, } \mathrm{m} / \mathrm{sec}^{2}\end{array}$ & supply, $\mathrm{t} /(\mathrm{h} \cdot \mathrm{m})$ \\
\hline 1 & etalon & 2,8 & 1000 & 15,2 & 6,7 \\
\hline 2 & etalon & 2,8 & 1000 & 15,2 & 6,7 \\
\hline 3 & etalon & 3,0 & 1000 & 15,2 & 6,7 \\
\hline 4 & etalon & 3,0 & 1000 & 15,2 & 6,7 \\
\hline 5 & etalon & 3,2 & 1000 & 15,2 & 6,7 \\
\hline 6 & etalon & 3,2 & 1000 & 15,2 & 6,7 \\
\hline 7 & etalon & 3,2 & 700 & 15,2 & 6,7 \\
\hline 8 & etalon & 3,2 & 700 & 15,2 & 6,7 \\
\hline 9 & etalon & 3,0 & 1000 & 18,9 & 6,7 \\
\hline 10 & etalon & 3,0 & 1000 & 18,9 & 6,7 \\
\hline 11 & new & $4,0 / 2,2$ & $130 / 850$ & 18,9 & 6,7 \\
\hline 12 & new & $4,0 / 2,2$ & $130 / 850$ & 18,9 & 6,7 \\
\hline 13 & new & $4,0 / 2,2$ & $100 / 850$ & 18,9 & 6,7 \\
\hline 14 & new & $4,0 / 2,2$ & $100 / 850$ & 18,9 & 6,7 \\
\hline 15 & new & $4,0 / 2,2$ & $100 / 850$ & 15,2 & 6,7 \\
\hline 16 & new & $4,0 / 2,2$ & $100 / 850$ & 15,2 & 6,7 \\
\hline 17 & new & $4,0 / 2,2$ & $130 / 850$ & 15,2 & 6,7 \\
\hline 18 & new & $4,0 / 2,2$ & $130 / 850$ & 15,2 & 6,7 \\
\hline 19 & new & $4,0 / 2,2$ & $130 / 850$ & 15,2 & 8,3 \\
\hline 20 & new & $4,0 / 2,2$ & $130 / 850$ & 15,2 & 8,3 \\
\hline 21 & new & $4,0 / 2,2$ & $150 / 850$ & 15,2 & 9,5 \\
\hline & & & & & \\
\hline
\end{tabular}


Table 3. Results of experiments on cleaning wheat seeds (continue of Table 2)

\begin{tabular}{|c|c|c|c|c|c|c|c|c|c|}
\hline \multirow{3}{*}{$\begin{array}{c}\text { №№ } \\
\text { pp }\end{array}$} & \multirow{3}{*}{$\begin{array}{l}\text { schem } \\
\text { e of } \\
\text { the } \\
\text { lattice } \\
\text { mill }\end{array}$} & \multicolumn{8}{|c|}{ Experiment Conditions } \\
\hline & & \multicolumn{2}{|c|}{ Fraction output, $\%$} & \multicolumn{3}{|c|}{ Content of waste, $\%$} & \multicolumn{3}{|c|}{$\begin{array}{l}\text { Completeness of separation of } \\
\text { waste }\end{array}$} \\
\hline & & $\begin{array}{l}\text { passage } \\
\text { sieve } B_{2}\end{array}$ & $\begin{array}{l}\text { passage } \\
\text { sieve } B_{1}\end{array}$ & $\begin{array}{c}\text { in } \\
\text { passage } \\
\text { sieve } \mathrm{B}_{2}\end{array}$ & $\begin{array}{l}\text { in retire } \\
\text { sieve } D_{2}\end{array}$ & $\begin{array}{l}\text { in the } \\
\text { purified } \\
\text { material }\end{array}$ & $\begin{array}{l}\text { upper } \\
\text { tier }\end{array}$ & lower tier & general \\
\hline 1 & etalon & 77,4 & 22,6 & 1,9 & 1,15 & 1,68 & 0,526 & 0,907 & 0,580 \\
\hline 2 & etalon & 77,8 & 22,2 & 1,83 & 1,05 & 1,72 & 0,542 & 0,915 & 0,500 \\
\hline 3 & etalon & 59,7 & 40,3 & 1,52 & 1,17 & 1,39 & 0,620 & 0,848 & 0653 \\
\hline 4 & etalon & 57,5 & 42,5 & 1,47 & 1,22 & 1,43 & 0,632 & 0,840 & 0,644 \\
\hline 5 & etalon & 20,2 & 79,8 & 0,83 & 1,44 & 1,40 & 0,793 & 0,697 & 0,649 \\
\hline 6 & etalon & 20,1 & 79,9 & 0,84 & 1,45 & 1,33 & 0,790 & 0,697 & 0,668 \\
\hline 7 & etalon & 53,2 & 46,8 & 1,79 & 1,10 & 1,53 & 0,553 & 0,835 & 0,617 \\
\hline 8 & etalon & 53,6 & 46,4 & 1,73 & 1,16 & 1,48 & 0,568 & 0,841 & 0,630 \\
\hline 9 & etalon & 48,2 & 51,8 & 1,41 & 0,95 & 1,18 & 0,648 & 0,859 & 0,705 \\
\hline 10 & etalon & 47,6 & 52,4 & 1,47 & 0,94 & 1,19 & 0,633 & 0,855 & 0,703 \\
\hline 11 & new & 34,5 & 65,5 & 0,62 & 0,89 & 0,80 & 0,845 & 0,845 & 0,800 \\
\hline 12 & new & 34,9 & 65,1 & 0,55 & 0,96 & 0,81 & 0,863 & 0,838 & 0,798 \\
\hline 13 & new & 49,7 & 50,3 & 1,21 & 0,86 & 1,04 & 0,698 & 0,878 & 0,740 \\
\hline 14 & new & 49,9 & 50,1 & 1,23 & 0,81 & 1,03 & 0,693 & 0,885 & 0,743 \\
\hline 15 & new & 51,8 & 48,2 & 1,23 & 0,75 & 0,98 & 0,693 & 0,895 & 0,755 \\
\hline 16 & new & 52,4 & 47,6 & 1,26 & 0,71 & 1,00 & 0,685 & 0,902 & 0,750 \\
\hline 17 & new & 38,6 & 61,4 & 0,90 & 1,08 & 1,01 & 0,775 & 0,819 & 0,748 \\
\hline 18 & new & 37,9 & 62,1 & 0,97 & 1,02 & 1,00 & 0,758 & 0,825 & 0,750 \\
\hline 19 & new & 52,7 & 47,3 & 1,59 & 0,91 & 1,27 & 0,903 & 0,865 & 0,683 \\
\hline 20 & new & 51,9 & 48,1 & 1,60 & 0,94 & 1,29 & 0,600 & 0,858 & 0,678 \\
\hline 21 & new & 54,6 & 45,4 & 1,93 & 1,05 & 1,54 & 0,518 & 0,840 & 0,615 \\
\hline
\end{tabular}

\section{Conclusion}

Experimental studies of purification of seeds on a two-tier the lattice mill made of polymeric materials was confirmed by the earlier revealed theoretical provisions on the possibility of increasing the efficiency of sieve seed cleaning machine work.

Installation in the grating instead of unloading sieve B1 composite polymer sieve, the first section of which is used for separation of the processed material into two parts, and the second to clean one of them from small waste, allows you to increase the completeness of the separation of waste from 0.71 up to 0.8 .

The performance of the sieve pan made of polymeric materials, executed under the new fractional scheme, in comparison with the production is higher by $24 \%$ with the same quality of seed cleaning.

At cleaning of wheat seeds the following parameters of composite polymer separators from SMPA B1 can be recommended:

- length of the first (fractional) section of the sieve $150 \ldots 200 \mathrm{~mm}$;

- the size of the holes of the sieve of the first section $-4,0 \ldots 4,5$;

- the length of the second (sorting) of a portion of a sieve $800-850 \mathrm{~mm}$;

- the size of the holes of the second portion of the sieve and 2.2...2.4 mm.

The experimental scheme of the lattice mill can be implemented in other designs, for example, installation in front of the sieve B1 standard size of additional polymer (fractional) sieve length $150 \ldots 200 \mathrm{~mm}$. 


\section{References}

1. Pehalsky I.A., Moskovsky M.N. Reduction of seed injury by sieves // Rural mechanic. 2015. № 9. C. 22-23.

2. Moskovsky M.N., Intensification of the process of separation of grain seeds in graincleaning aggregates. / Dissertation PHD 05.20.01 /Rostov-on-Don, 2005.

3. Moskovsky M.N., Muratov D.K., Boyko A.A., Experimental studies of the functioning sieve cleaning of polymer material of UHMW PE in departments of combine harvesters // Engineering journal of electronic science and innovation Magazine. - 2016 No1. 